\title{
Chemical, Petrographic, and K-Ar Age Data to Accompany Reconnaissance Geologic Strip Map from Kingman to South of Bill Williams Mountain, Arizona
}

\author{
Barbara Arney \\ Fraser Goff \\ Andrea C. Eddy
}

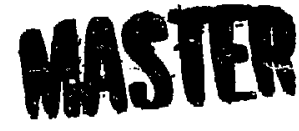

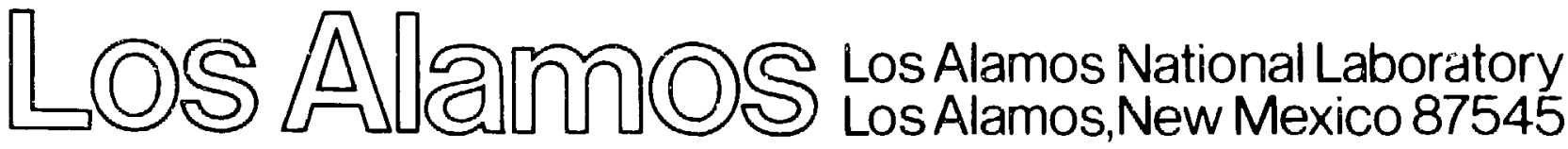


CHEMICAL, PETROGRAPHIC, AND K-Ar Age DATA to ACCOMPANY RECONNAISSANCE GEOLOGIC STRIP MAP FROM RINGMAN TO SOUTH OF BILL WILLIAMS MOUNTAIN, ARIZONA

by

Barbara Arney, Fraser Goff, and Andrea C. Eddy

\begin{abstract}
As part of a reconnaissance mapping project, 40 chemical analyses and 13 potassium-argon age dates were obtained for Tertiary volcanic and Precambrian granitic rocks between Kingman and Bill Williams Mountzin, Arizona. The dated volcanic rocks range in age from $5.5 \pm 0.2 \mathrm{Myr}$ for basalt in the East Juniper Mountains to about 25 Myr for a biotite-pyroxere andesite. The date for Picacho Butte, a rhyodacite in the the Mt. Floyd volcanic field, was $9.8 \pm$ 0.07 Myr, making it the oldest rhyodacite dome in that volcanic field. Dated rocks in the Fort Rock area range from 20.7 to $24.3 \mathrm{Myr}$. No ages were obtained on the Precambrian rocks. Compositionally, the volcanic rocks analyzed range from alkali basalt to rhyolite, but many rocks on the western side of the map area are unusually potassic. The granites chosen for analysis include syenogranite from the Hualapai Mountains, a muscovite granite from the the Picacho Butte area, and two other granites. The chemical and K-Ar age data and petrographic descriptions included in this report accompany the reconnaissance geologic strip map published as LA-9202-MAP by Goff, Eddy, and Arney.
\end{abstract}

\title{
I. INTRODUCTION
}

As part of the national Hot Dry Rock evaluation program, we mapped a strip about 10 to $15 \mathrm{~km}$ wide and $160 \mathrm{kIn}$ long from Kingman to just south of Bill Williams Mountain in Arizona (Fig 1.) The purpose was to obtain a reconnaissance map which would follow the trace of a magnetotelluric (MT) survey being done by Los Alamo; National Laboratory across the transition zone between the Basin and Range and the Colorado Plateau tectonic provinces (Aiken and Ander 1981; Ander and Furgerson 1982; Ander 1983). The color strip map has been published separately by Los Alamos National Laboratory (Goff et al. 1983). 


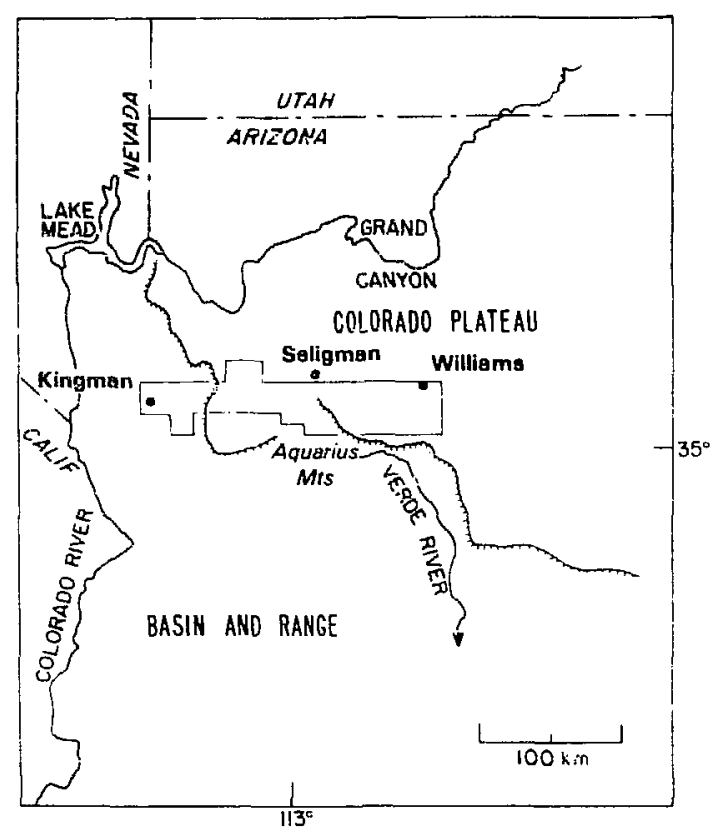

Fig. 1 .

Map showing southwest boundary between Basin and Range and Colorado Plateau Provinces and location of area covered by strip map.

Considerable geochemical data on the volcanic rocks and limited data on Precambrian rocks of the area were obtained and are presented in this report. Petrographic descriptions of rocks that were analyzed or dated are given in the Appendix.

The map crosses three geoiogic zones. The western part of the map crosses the eastern edge of the Basin and Range Province. This zone is characterized by mafic volcanism and block faulting along north-trending faults. The eastern part of the map crosses the Mt. Floyd volcanic area, part of the greater San Francisco Peaks volcanic field. In this zone basalts and basaltic andesite overlie Paleozoic sedimentary rocks of the Colorado plateau. In between, from Tir Mountain on the west to the C...no Vailey on the east, the map crosses $a$ tectonic transition zone between the Basin and Range and the Colorado Plateau. On the west side of this middle zone, Tertiary volcanics of basaltic to rhyolitic composition overlie Precambrian granite and gneiss. The thickness of Paleozoic sedimentary rocks increases to the east across this zone, and the volcanic rocks overlie increasingly younger paleozoic rocks. The structure is dominated by northwest-trending normal faults, with displacement generally down to the west. Stratigraphic relations and the age dates presented in Table I show that the ages of the volcanic rocks, particularly the basaltic rocks, generally decrease to the east across the transition zone.

\section{WESTERN ZONE}

Volcanic rocks of the Kingman area include olivine basalt, basaltic andesite and the Peach Springs Tuff, a rhyolite ash-flow tuff that probably originated northwest of Kingman and flowed eastward for more than a hundred kilometers (Young and Brennan 1974). There is uncertainty about the age 
POTASSIUM-ARGON DATES ${ }^{\text {TABLE I }}$ OBTAINED FROM VOLCANIC ROCKS

MAPPED BETWEEN KINGMAN AND BILL WILLIAMS MOUNTAIN, ARIZONA

\begin{tabular}{|c|c|c|c|c|c|c|c|c|c|c|}
\hline Sample & Location & $\begin{array}{l}\text { Map } \\
\text { Symbol }\end{array}$ & $\begin{array}{l}\text { Rack } \\
\text { Type }\end{array}$ & Materid! & $\mathbf{2 k}$ & $\begin{array}{l}\text { Moles } \\
\text { Radiogenic } \\
\text { Argon }\end{array}$ & $\begin{array}{l}\text { Radiogenic } \\
\text { Argon }\end{array}$ & Aye, & Myr & Conmelit s \\
\hline$\frac{\text { Volcanic ro }}{1.778-59}$ & 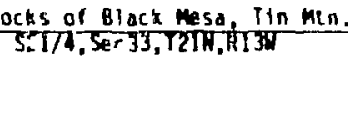 & Thed 1 & $\begin{array}{l}\frac{1 / 2}{\text { Quad }} \text { Quaditic } \\
\text { Dacite }\end{array}$ & ic & 6.11 & $4.33 \times 10^{-11}$ & 48.0 & $20.5 \pm$ & 1.0 & $\begin{array}{l}\text { Whit was mapped as overlyiny Yeach Sprinys } \\
\text { iuff, but tulf may onl, lill a swale on } \\
\text { sloe of dacite flow. }\end{array}$ \\
\hline 2. $178-64$ & $\operatorname{SE} 1 / 4, \operatorname{Sec} 17,[21 \mathrm{H}, \mathrm{R} 1 \mathrm{JH}$ & Tbd & Andesite & $W / H$ & 2.28 & $2.83 \times 10^{-11}$ & 27.9 & $11.8 \pm$ & u.7 & Andesice tlow overlies Peach Sprinys Iult. \\
\hline$\frac{\text { Yolcantc ror }}{3.778-72}$ & 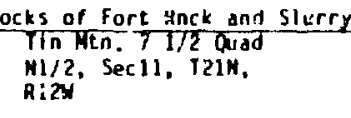 & $\frac{1 \text { L line }}{\text { TrhT }}$ & Hol Lat ite & Bio & 3.57 & $3.08 \times 10^{-11}$ & 34.2 & 24.32 & u.8 & $\begin{array}{l}\text { Latite dowe overlies marliblenae-bedriny } \\
\text { dikali-olivine basalt. }\end{array}$ \\
\hline 4. F 78-85 & $\begin{array}{l}\text { Bull Springs } 71 / 2 \text { quad } \\
\text { Lat. } 35^{\circ} 12^{\prime} \text {, Long. } 113^{\circ} 25^{\prime}\end{array}$ & Irbi & $\begin{array}{l}\text { Ho! } 011 \text { y } \\
\text { Basale }\end{array}$ & $W / R$ & 1.98 & $4.43 \times 10^{-11}$ & $3 / .8$ & 22.01 & 0.7 & $\begin{array}{l}\text { Hdsalt dike cuts fort kock ireek Iulf (ot } \\
\text { fuls, (y)J). }\end{array}$ \\
\hline 5. BA7R-73 & $\begin{array}{l}\text { Ft. Rock ME I 1/2 Ouad } \\
\text { Lat. } 35^{\circ} 14^{\circ} \text {, Long. } 111^{\circ} 20^{\prime}\end{array}$ & Isba & $\begin{array}{l}\text { Basaltic } \\
\text { Andesite }\end{array}$ & $H / R$ & 3.10 & $5.58 \times 10^{-11}$ & 66.0 & $2 n+1$ & u.t & $\begin{array}{l}\text { Flows were napped as equivalent to ones that } \\
\text { overlie jedch Springs Jutf to nortl:; these } \\
\text { flows way incertinger with Peach Springs } \\
\text { Iuff. }\end{array}$ \\
\hline$\frac{\text { Yolcanic roc }}{\text { 6. BR78-5?h }}$ & $\begin{array}{l}\text { ocks of Squax Peak } \\
\text { Cross ALn. HE } 71 / 2 \text { Ouad } \\
\text { Lat. } 35^{\circ} 12^{\circ} \text {, Long. } 113^{\circ} 6^{\prime \prime}\end{array}$ & Tpob & $\begin{array}{l}\text { Oifrine } \\
\text { Basalt }\end{array}$ & $\omega / 2$ & 0.63 & $5.19 \times 10^{-12}$ & 14.2 & $9.0 \pm$ & 1.2 & $\begin{array}{l}\text { Youngest flow in stack erupted from squas } \\
\text { Peak; flows generally outcrop to east and are } \\
\text { cut by numerous nomeal faults. }\end{array}$ \\
\hline$\frac{\text { Yolcanic rac }}{7 . \text { BhtB-82 }}$ & $\begin{array}{l}\text { ocks of East Juniper Ktns.. } \\
\text { Center, Sec21, T21W, R5W }\end{array}$ & Turkey & $\begin{array}{l}\text { Canjon HE ? } \\
\text { Olivine } \\
\text { Basalt }\end{array}$ & $\frac{21 / 2 \text { Quad }}{W / R}$ & ${ }_{i .50}$ & $1.44 \times 10^{-12}$ & 11.5 & $3.5=$ & 0.2 & 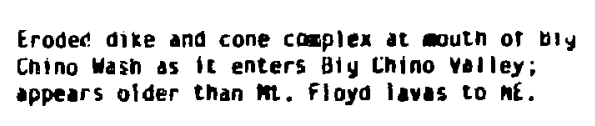 \\
\hline 8. $B A 78-90$ & Center, Secl, TzOK, R6H & Tjob & $\begin{array}{l}\text { Olivine } \\
\text { Basalt }\end{array}$ & $W / R$ & 1.16 & $6.09 \times 10^{-12}$ & 45.18 & 6.21 & 0.3 & $\begin{array}{l}\text { Basalt flow overlies ledwall Lienstone; } \\
\text { adjacent to BA7t- } 72 \text { mentioned below. }\end{array}$ \\
\hline$\frac{\text { Mt. Floyd vo }}{9 . \text { Fro-113 }}$ & $\frac{\text { Volcanic Field, Picacho Butt }}{\text { ciest of Picachio Butto }}$ & te $71 / 2$ & $\begin{array}{l}12 \text { Quad } \\
\text { HDT Rhyo- } \\
\text { dactie }\end{array}$ & Hol & 0.87 & $9.02 \times 10^{-12}$ & 19.2 & 9.81 & 0.7 & $\begin{array}{l}\text { Eroded rhyodacile dowe surrounded by basalt } \\
\text { flows of mt. Floyd rolcanic tield. }\end{array}$ \\
\hline$\frac{\text { Andesites of }}{\text { IIT. F78-88 }}$ & $\begin{array}{l}\text { of Turkey Canyon } \\
\text { Ft. Rock NE T } 1 / 2 \text { quad } \\
\text { Lat. } 35^{\circ} 11^{\prime} \text {, Long. } 113^{\circ} 18^{\prime}\end{array}$ & Ttc & $\begin{array}{l}\text { Bio-Pyr } \\
\text { Mndesite }\end{array}$ & Bio & 6.61 & $5.26 \times 10^{-11}$ & 61.0 & 22.41 & 0.8 & $\begin{array}{l}\text { Andesite dawe dind flowoverldin and surrounded } \\
\text { by younger basale flows. }\end{array}$ \\
\hline 11. BA78-72 & $\begin{array}{l}2 \text { Iurkey Can, UE } ? \text { I/2 Ouad } \\
\text { SE } 1 / 4, \operatorname{Sec} 12, \text { I2OK, A6W }\end{array}$ & Ttc & $\begin{array}{l}\text { Bla-Pyr } \\
\text { Andesite }\end{array}$ & $\begin{array}{c}\text { Bta } \\
W ; R\end{array}$ & $\begin{array}{l}7.16 \\
1.16 \\
4.10\end{array}$ & $\begin{array}{l}8.23 \times 10^{-1} \\
6.62 \times 10^{-11} \\
2.12 \times 10^{-11}\end{array}$ & $\begin{array}{l}21.0 \\
60.1\end{array}$ & $\begin{array}{l}36.1: \\
32.0: \\
24.4:\end{array}$ & $\begin{array}{l}1.2 \\
2.0 \\
1.2\end{array}$ & 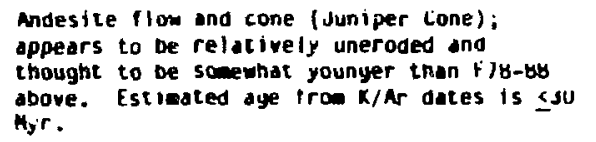 \\
\hline
\end{tabular}


of the Peach Springs Tuff. Young and Brennan (1974) report dates of 16.9 and 18.3 Myr, but believe it to be a single cooling unit. Because this unit is the primary stratigraphic marker in Tertiary volcanic rocks of this region, an age of $17.6 \mathrm{Myr}$ is used in this report (Young and 8renran 1974). In the Kingman area, the Peach Springs Tuff overlies basalts and basaltic andesites that young (1985, oral commun.) believes are not much older than the tuff. Many of the basaltic andesites and andesites (Table II) contain resorbed quartz xenocrysts. The volcanic rocks directly overlie Precambrian granite, schist, and gneiss. Two K-Ar dates $(20.5 \pm 1.0$ and $13.8 \pm 0.7$ Myr $)$ were obtained on volcanic rocks of Black Mesa (southeast of the Peacock Mountains and west of the cottonwood Cliffs). These rocks range from dacite to alkali basalt but are chemically unique to Black Mesa and not representative of volcanic rocks in the western zone of the mapped area.

\section{TRANSITION ZONE}

Based on potassium content and age dates, the volcanic rocks of the transition zone form three groups (Figs. 2 and 3): a high-potasium group on the west side (Tin Mountain-Cross Mountain), a jounger low-potassium group toward the east (Juniper Mountains-Mt. Floyd volcanics), and biotite-pyroxene andesites (Turkey Canyon Andesite). This last group pre dates Basin and Range faulting and most transition zone volcanism and forms a chain of plugs, domes, and flows that crosses the present $\mathrm{N}-\mathrm{NW}$ structural trends (Fig. 2). The groups are not very distinct in their major element concentrations aside from potassium (Fig. 4). The biotite-pyroxene andesites do not appear to be related to the younger volcanics in chemistry or in time. The $K-A r$ dates determined on biotite separates from two of the andesites are 22.4 Myr and 32-36 Myr (two dates). A whole-rock date on the last sample was 4.4 Myr, considerably younger than the dates from biotite separates of that rock but a more reasonable age in light of the chemical similarities cf this group of lavas.

The only major occurrence of silicic rocks in the entire mapped area is in the Fort Rock area, just east of Tin Mountain. Volcanic rock types in the Fort Rock area include pyroxene andesites, hornblende andesites, dacites and latites as well as basalt, basaltic andesite, and two rhyolitic tuff units. One latite dome on the west edge of the area contains a rare occurrence of scapolite phenocrysts (Goff et al. 1982). The most easterly known outcrop of 
TABLE II

CHEMICAL ANALYSES IN WEIGHT-PERCENT OF 45 VDLCANIC AND GRANITIC ROCKS BETWEEN KINGMAN AND BILL WILLIAMS MOUNTAIN, ARIZONA

\begin{tabular}{|c|c|c|c|c|c|c|c|c|c|c|c|}
\hline \multirow[b]{2}{*}{ Rock Type } & \multicolumn{3}{|c|}{ kingman Area } & \multirow{2}{*}{$\frac{\text { Hualapa i Mtns. }}{\text { Syenogranite }}$} & \multirow{2}{*}{$\begin{array}{l}\text { Peacork Mtons. } \\
\begin{array}{l}\text { olivine } \\
\text { Andesite }\end{array}\end{array}$} & \multicolumn{4}{|c|}{ Black Mesa Area } & \multicolumn{2}{|c|}{ Peacn Springs Tuff } \\
\hline & $\begin{array}{l}\text { Olivine } \\
\text { Basale }\end{array}$ & $\begin{array}{l}\text { Basalcic } \\
\text { Andesite }\end{array}$ & $\begin{array}{l}\text { Basaltic } \\
\text { Andesite }\end{array}$ & & & Andesite & $\begin{array}{l}\text { Biotite- } \\
\text { olivine } \\
\text { Andesite }\end{array}$ & $\begin{array}{l}\text { Por- } \\
\text { phyritic } \\
\text { Oacite }\end{array}$ & Granite & $\begin{array}{l}\text { Black } \\
\text { Mesa }\end{array}$ & $\begin{array}{l}\text { Aquarius } \\
\text { Mtns. }\end{array}$ \\
\hline Sample No. & $F 78-50$ & F78-45 & F78-49 & $F 78-54$ & $6-78-A C E-16$ & $F 78-64$ & $F 78-61$ & F78-59 & $F 78-60$ & $F 78-63$ & Fuis, 1973 \\
\hline $\mathrm{SiO}_{2}$ & 44.33 & 56.26 & 59.72 & $7 ! .02$ & 54.91 & 55.17 & 57.96 & 65.61 & 77.25 & 74.55 & 67.0 \\
\hline $\mathrm{TiO}_{2}$ & 2.75 & 0.96 & 1.18 & 0.19 & 1.33 & 0.98 & 0.94 & 0.56 & 0.12 & 0.25 & 0.21 \\
\hline $\mathrm{Al}_{2} \mathrm{O}_{3}$ & 9.97 & 14.92 & 15.25 & 14.99 & 11.76 & 18.94 & 13.39 & 15.94 & 12.22 & 13.48 & 13.9 \\
\hline $\mathrm{Fe}_{2} \mathrm{O}_{3}$ & 2.46 & 2.76 & 3.05 & 0.83 & 3.18 & 3.30 & 2.89 & 1.68 & 0.37 & 0.59 & 1.0 \\
\hline $\mathrm{Fe}^{\mathrm{b}}$ & 8.85 & 3.16 & 3.48 & 0.95 & 3.54 & 3.78 & 3.30 & 1.92 & 0.43 & 0.68 & 0.22 \\
\hline Mgo & 13.76 & 4.78 & 4.31 & 0.35 & 6.66 & $: 39$ & 5.76 & 2.42 & 0.23 & 0.42 & 1.3 \\
\hline CaI & 10.13 & 7.16 & 5.82 & 0.35 & 7.60 & 5.92 & 5.98 & 3.90 & 0.31 & 0.96 & 1.4 \\
\hline Mno & 0.17 & 0.09 & 0.09 & 0.02 & 0.10 & 0.10 & 0.09 & 0.06 & 0.03 & 0.07 & 0.08 \\
\hline $\mathrm{Na}_{2} \mathrm{O}$ & 3.31 & 3.42 & 4.03 & 3.38 & 2.74 & 4.61 & 2.63 & 4.05 & 2.82 & 3.54 & 3.6 \\
\hline$x_{2}{ }^{0}$ & 1.30 & 3.10 & 3.06 & 6.80 & 5.96 & 2.37 & 5.80 & 3.56 & 5.11 & 5.37 & 4.4 \\
\hline $\mathrm{P}_{2} \mathrm{O}_{5}$ & 0.78 & 0.53 & 0.35 & 0.05 & 0.90 & is. 42 & 0.70 & 0.22 & 0.03 & 0.04 & 0.04 \\
\hline $\mathrm{H}_{2} \mathrm{Ol}+1$ & & 1.05 & & & & 0.17 & & & & & 3.53 \\
\hline $\mathrm{H}_{2} \mathrm{O}(-)$ & & 0.33 & & & & 0.18 & & & & & 3.15 \\
\hline $\mathrm{CO}_{2}$ & & 0.51 & & & & 0.27 & & & & & 0.44 \\
\hline $8 \mathrm{a} 0$ & & 0.17 & & & & 0.13 & & & & & \\
\hline Sro & & 0.13 & & & & 0.10 & & & & & \\
\hline Total & 97.81 & 99.43 & 100.34 & 99.43 & 93.78 & 99.33 & 99.44 & 99.92 & 98.92 & 99.95 & 100.17 \\
\hline \multicolumn{12}{|c|}{ CIPH Norms (wt b) } \\
\hline 0 & & 6.69 & 8.32 & 22.78 & & 3.04 & $3.7 B$ & 17.15 & 40.21 & 30.62 & 25.60 \\
\hline Co & & & & .64 & & & & & 1.56 & .19 & 1.69 \\
\hline or & 7.68 & 13.32 & 18.08 & 40.18 & 35.22 & 14.00 & 34.27 & 21.04 & 30.20 & 31.73 & 26.00 \\
\hline$A D$ & 10.60 & 28.94 & 34.10 & 28.60 & 23.19 & 39.01 & 22.25 & 34.27 & 23.86 & 29.95 & 30.46 \\
\hline An & 8.51 & 16.20 & 14.48 & 2.89 & 2.19 & 23.99 & 7.60 & 14.80 & 1.34 & 4.50 & 3.90 \\
\hline $\mathrm{Ne}$ & 9.43 & & & & & & & & & & \\
\hline$D i$ & 29.28 & 10.02 & 9.60 & & 23.36 & 1.15 & 13.83 & 2.47 & & & \\
\hline Hy & & 9.29 & 8.28 & 1.65 & .64 & 9.43 & 10.11 & 6.18 & .91 & 1.52 & 3.24 \\
\hline 01 & 21.72 & & & & 4.97 & & & & & & \\
\hline$M \mathrm{t}$ & 3.57 & 4.00 & 4.42 & 1.20 & 4.61 & 4.78 & 4.19 & 2.44 & .54 & .86 & .36 \\
\hline $11 m$ & 5.22 & 1.82 & 2.24 & .36 & 2.53 & 1.86 & 1.79 & 1.06 & .23 & .47 & .40 \\
\hline $\mathrm{Hm}$ & & & & & & & & & & & .75 \\
\hline Ap & 1.85 & 1.26 & .83 & .12 & 2.13 & .99 & 1.66 & .52 & .07 & .09 & .09 \\
\hline $\mathrm{Cc}$ & & 1.39 & & & & .61 & & & & & 1.00 \\
\hline
\end{tabular}


TABLE II (cont)

\begin{tabular}{|c|c|c|c|c|c|c|c|c|}
\hline \multirow[b]{2}{*}{ Rack Type } & \multicolumn{3}{|c|}{ Fort Rock Creek Tuff ${ }^{C}$} & \multicolumn{5}{|c|}{ Fort Rock Ared } \\
\hline & $\begin{array}{l}\text { Lithic } \\
\text { Tuff }\end{array}$ & $\begin{array}{l}\text { Massive } \\
\text { Tufi }\end{array}$ & $\begin{array}{l}\text { Bedded } \\
\text { Iuff }\end{array}$ & $\begin{array}{l}\text { Olivinte } \\
\text { Basalt }\end{array}$ & $\begin{array}{l}\text { Olivine } \\
\text { Basalt }\end{array}$ & $\begin{array}{l}\text { Olivine } \\
\text { Basalt }\end{array}$ & $\begin{array}{l}\text { Pyroxene } \\
\text { Andesite }\end{array}$ & $\begin{array}{l}\text { Hornblende } \\
\text { Andesite }\end{array}$ \\
\hline Sample No. & $6-7 . A-A C E-22$ & Furs, 1973 & Fuis, 1973 & $6-78-A C E-30 C$ & Fut, 1973 & $b-7 t-A C E-3 b$ & Fuis, 1973 & $F 7 y-8:$ \\
\hline $\mathrm{SiO}_{2}$ & 69.38 & 76.92 & 70.94 & 48.71 & 50.0 & 52.14 & 54.9 & 37.81 \\
\hline $\mathrm{TiO}^{5}$ & 0.36 & $0.2 \mathrm{H}$ & 0.26 & 1.69 & 1.2 & $1 . f i 2$ & 1.25 & 1.13 \\
\hline $\mathrm{Al}_{2} \mathrm{O}_{3}$ & 13.68 & 11.82 & 15.04 & 15.98 & 14.7 & $17.4 t$ & 15.3 & 17.28 \\
\hline $\mathrm{Fe}_{2} \mathrm{O}_{3}$ & 1.05 & 1.53 & 1.64 & 2.17 & 6.9 & 2.17 & 5.1 & $3.6 \%$ \\
\hline $\mathrm{FeO}^{\mathrm{b}}$ & 1.20 & 0.39 & 0.55 & 7.83 & 1.2 & 7.83 & 2.16 & 4.15 \\
\hline MgO & 3.71 & 1.31 & 1.27 & 5.51 & 4.6 & 4.96 & 5.8 & 3.19 \\
\hline CaO & 3.97 & 2.74 & 2.33 & 9.64 & 0.7 & 9.54 & 6.7 & 6.41 \\
\hline Mno & 0.08 & 0.08 & 0.11 & 0.16 & 0.13 & 0.16 & 0.10 & 0.09 \\
\hline $\mathrm{Na}_{2} \mathrm{O}$ & 2.62 & $1.8 E$ & 3.60 & 3.70 & 2.9 & 4.25 & 3.8 & 4.01 \\
\hline$K_{2} 0$ & 3.83 & 2.95 & 4.13 & 1.64 & 3.1 & 0.77 & 2.4 & 2.84 \\
\hline $\mathrm{P}_{2} \mathrm{O}_{5}$ & 0.12 & 0.12 & 0.08 & 1.11 & 0.99 & 0.38 & 0.44 & 0.34 \\
\hline $\mathrm{H}_{2} \mathrm{O}(+)$ & & & & & 1.6 & & 1.05 & \\
\hline $\mathrm{H}_{2} \mathrm{D}(-)$ & & & & & 1.8 & & 0.96 & \\
\hline $\begin{array}{l}\mathrm{CO}_{2} \\
\mathrm{BaO}\end{array}$ & & & & & 1.6 & & $<0.05$ & \\
\hline \multicolumn{9}{|l|}{ Sro } \\
\hline Total & 100.00 & 100.00 & 100.00 & $9 B .44$ & 99.42 & 101.28 & 100.0 & 100.87 \\
\hline \multicolumn{9}{|c|}{ CIPW Norms (wt b) } \\
\hline$Q$ & 26.38 & 47.33 & 27.53 & 4.80 & & 4.61 & 6.65 & \\
\hline Co & & .87 & .60 & & & & & \\
\hline or & 22.53 & 17.43 & 29.41 & 9.69 & 18.32 & 4.55 & 14.18 & 16.78 \\
\hline An & 22.17 & 15.74 & 30.46 & 30.45 & 24.54 & 35.96 & $32=15$ & 33.93 \\
\hline An & 14.26 & 12.81 & 11.04 & 22.15 & $17.9 \overline{4}$ & 26.29 & 17.60 & 20.76 \\
\hline Me & & & & .47 & & & & \\
\hline Di & 3.66 & & & 13.48 & 6.73 & 15.26 & 9.93 & 7.08 \\
\hline $\mathrm{Hy}$ & 8.42 & 3.26 & 3.16 & & 8.34 & 4.06 & 9.84 & 7.48 \\
\hline$u$ & & & & 12.58 & & 8.06 & & \\
\hline Mt & 1.52 & .71 & 1.38 & 3.15 & .81 & 3.15 & 3.67 & 5.25 \\
\hline $11 \mathrm{~m}$ & .68 & .53 & .49 & 3.21 & 2.28 & 3.08 & 2.37 & 2.15 \\
\hline $\mathrm{Hm}$ & & 1.04 & .74 & & 6.34 & & 2.57 & \\
\hline$A p$ & .28 & .28 & .19 & 3.34 & 2.34 & .90 & 1.04 & .81 \\
\hline$r=$ & & & & & 3.64 & & & \\
\hline
\end{tabular}


TABLE II (cont)

Fort Rock Ared

North Fort Rack Ared

\begin{tabular}{|c|c|c|c|c|c|c|c|c|c|}
\hline Rock Type & $\begin{array}{l}\text { Basaltic } \\
\text { Andesite }\end{array}$ & $\begin{array}{l}\text { Hornolende } \\
\text { Andesite }\end{array}$ & $\begin{array}{c}\text { Harnblende } \\
\text { Latite }\end{array}$ & $\begin{array}{l}\text { Porphyritic } \\
\text { Rhyoddcite }\end{array}$ & Granite & $\begin{array}{l}\text { Olivine } \\
\text { Basalt }\end{array}$ & $\begin{array}{l}\text { Basaltic } \\
\text { Andesite }\end{array}$ & $\begin{array}{l}\text { Basalt if } \\
\text { Andesite }\end{array}$ & $\begin{array}{l}\text { Hypersthene } \\
\text { Andesite }\end{array}$ \\
\hline Cample No. & $6-78-A C E-37$ & BA78- 14 & F $78-74$ & $B A / B-30$ & $F / 8-87$ & $F 78-83$ & BA78-37 & F78-79 & $B A 7 B-89$ \\
\hline $\sin _{2}$ & 58.26 & 62.62 & 68.48 & 71.14 & 74.46 & 53.00 & 52.85 & 56.44 & 60.54 \\
\hline $\mathrm{TiO}_{2}$ & 1.33 & 0.65 & 0.33 & 0.36 & 0.24 & 1.00 & 1.48 & 1.05 & 0.90 \\
\hline $\mathrm{Al}_{2} \mathrm{O}_{3}$ & 17.27 & 17.88 & 17.71 & 14.48 & 14.67 & 13.34 & 18.26 & 14.63 & 14.42 \\
\hline $\mathrm{Fe}_{2} \mathrm{O}_{3}$ & 3.16 & 2.29 & 1.29 & 1.16 & 0.69 & 1.60 & 1.80 & 3.21 & 2.78 \\
\hline$F e 0^{\circ}$ & 4.28 & 2.62 & 1.47 & 1.33 & 0.79 & 5.75 & 6.48 & 3.68 & 3.19 \\
\hline $\mathrm{Mg} 0$ & 2.22 & 1.15 & 0.93 & 1.18 & 0.65 & 10.33 & 4.48 & 6.69 & 5.06 \\
\hline $\mathrm{CaO}$ & 5.91 & 4.05 & 2.48 & 2.61 & 2.53 & 7.16 & 9.22 & 7.49 & 5.51 \\
\hline $\mathrm{MnO}$ & 0.12 & 0.08 & 0.06 & 0.04 & 0.04 & 0.22 & 0.13 & 0.11 & 0.08 \\
\hline $\mathrm{Na}_{2} \mathrm{O}$ & 4.29 & 4.39 & 4.36 & 3.98 & 3.82 & 2.72 & 3.83 & 3.47 & 3.48 \\
\hline$k_{2} 0$ & 2.96 & 4.14 & 4.14 & 3.38 & 3.35 & 2.37 & 1.28 & 3.09 & 3.81 \\
\hline $\mathrm{P}_{2} \mathrm{O}_{5}$ & 0.47 & 0.44 & 0.21 & 0.13 & 0.07 & 0.40 & 0.41 & 0.40 & 0.31 \\
\hline $\mathrm{H}_{2} \mathrm{O}(+)$ & & & & & & 0.01 & & & \\
\hline $\mathrm{H}_{2} \mathrm{O}(-)$ & & & & & & 0.21 & & & \\
\hline $\mathrm{CO}_{2}$ & & & & & & 1.28 & & & \\
\hline 800 & & & & & & 0.12 & & & \\
\hline Sro & & & & & & 0.12 & & & \\
\hline Totai & 100.27 & 100.31 & 101.45 & 99.79 & 101.31 & 99.53 & 100.22 & 100.26 & 100.08 \\
\hline
\end{tabular}

CIPW Norms (wt g)

\begin{tabular}{|c|c|c|c|c|c|c|c|c|c|}
\hline 0 & 7.38 & 11.26 & 20.62 & 27.72 & 32.97 & & & 2.54 & 9.00 \\
\hline Co & & & 2.65 & & .33 & & & & \\
\hline or & 17.49 & 24.46 & 24.46 & 19.97 & 19.80 & 14.00 & 7.56 & 18.26 & 22.51 \\
\hline$A D$ & 36.30 & 37.15 & 36.89 & 33.68 & 32.32 & 23.02 & 32.41 & 29.36 & 23.45 \\
\hline An & 19.12 & 16.85 & 10.93 & 11.66 & 12.09 & 17.19 & 28.85 & 15.22 & 12.47 \\
\hline \multicolumn{10}{|l|}{ Ne } \\
\hline Di & 5.80 & .30 & & .35 & & 6.53 & 11.64 & 15.27 & 10.16 \\
\hline Hy & 5.98 & 4.71 & 3.52 & $3.7 \mathrm{~d}$ & 2.18 & 30.29 & 12.64 & 12.04 & 10.02 \\
\hline 01 & & & & & & .09 & .75 & & \\
\hline Mt & 4.58 & 3.32 & 1.87 & 1.68 & 1.00 & 2.32 & 2.61 & 4.65 & 4.03 \\
\hline $11 \mathrm{~m}$ & 2.53 & 1.23 & .63 & .68 & .46 & 1.90 & 2.81 & 1.99 & 1.71 \\
\hline \multicolumn{10}{|l|}{$\mathrm{Hm}$} \\
\hline Ap & 1.11 & 1.04 & .50 & .31 & .17 & .95 & .97 & .95 & .73 \\
\hline Cc & & & & & & 2.91 & & & \\
\hline
\end{tabular}


TABLE II (cont)

\begin{tabular}{|c|c|c|c|c|c|c|c|c|c|c|c|c|}
\hline \multirow[b]{2}{*}{ Rock Type } & \multicolumn{3}{|c|}{ Cross MLn. Ared } & \multicolumn{3}{|c|}{ Squaw 'Pti' Area } & \multirow{2}{*}{$\frac{\begin{array}{c}\text { Henry Brown } \\
\text { Mtn. }\end{array}}{\begin{array}{c}\text { High-Alumina } \\
\text { Basalt }\end{array}}$} & \multirow{2}{*}{$\begin{array}{l}\text { Turkey } \\
\text { Sinrices. } \\
\text { Pyroxtene } \\
\text { Andesile }\end{array}$} & \multicolumn{2}{|c|}{ Canyon Andesite } & \multicolumn{2}{|c|}{$\begin{array}{c}\text { East Juniper Mits. } \\
\text { arrea }\end{array}$} \\
\hline & $\begin{array}{l}\text { Basaltic } \\
\text { Andesite }\end{array}$ & $\begin{array}{l}\text { Olivine } \\
\text { Andesite }\end{array}$ & $\begin{array}{l}\text { Biocite } \\
\text { Andesite }\end{array}$ & $\begin{array}{l}\text { Ulivine } \\
\text { Basalt }\end{array}$ & $\begin{array}{l}\text { Basaltic } \\
\text { Andesice }\end{array}$ & $\begin{array}{l}\text { hornblende } \\
\text { indesite }\end{array}$ & & & $\begin{array}{l}\text { Biotite- } \\
\text { Pyroxene } \\
\text { Andesite }\end{array}$ & $\begin{array}{l}\text { Biotite- } \\
\text { Fyroxene } \\
\text { Andesite }\end{array}$ & $\begin{array}{l}\text { Fyroxene } \\
\text { Basalt }\end{array}$ & $\begin{array}{l}\text { Sasaltic } \\
\text { Andesite }\end{array}$ \\
\hline Sample Na. & $F 78-89$ & 8-/8-ACE-21 & F $70-102$ & BA78-52 & $8078-59$ & $2478-63$ & $8-78-A C E-14$ & F $78-88$ & $F 78-98$ & BA78-71 & $3478-82$ & $B A 78-76$ \\
\hline $\mathrm{SiO}_{2}$ & 54.92 & 56.01 & 59.73 & 50.70 & 58.10 & 59.65 & 48.29 & 60.33 & 56.34 & 63.42 & 54.34 & 59.22 \\
\hline $\mathrm{TiO}_{2}$ & 1.08 & 0.87 & $1.0 ?$ & 1.32 & 0.92 & 0.84 & $2.1 n$ & $\therefore .07$ & $1.2 C$ & 0.72 & 1.46 & .96 \\
\hline $\mathrm{Al}_{2} \mathrm{O}_{3}$ & 13.31 & 13.60 & 13.84 & 15.61 & 15.52 & 11.06 & 17.75 & 14.00 & 14.91 & 14.53 & 16.71 & $16.0 n$ \\
\hline $\mathrm{Fe}_{2} \mathrm{O}_{3}$ & 3.89 & 3.60 & 2.90 & 2.20 & $3.6 j$ & 2.94 & 2.46 & 2.86 & 3.12 & 2.32 & $3.6 i$ & 2.91 \\
\hline $\mathrm{Fen}$ ? & 4.45 & 4.13 & 3.33 & 7.92 & 4.15 & 3.37 & 8.84 & 3.28 & 3.57 & 2.55 & 4.20 & 3.34 \\
\hline $\mathrm{MgO}$ & 8.93 & 9.10 & 5.55 & 8.69 & 5.58 & 4.18 & 5.56 & 3.22 & 4.05 & 3.32 & $5.0:$ & 3.83 \\
\hline $\mathrm{CaO}$ & 6.45 & 6.60 & 6.13 & 9.33 & 6.84 & 5.87 & 9.53 & 5.69 & 6.30 & 4.85 & $5 \geq 1$ & 6.35 \\
\hline $\operatorname{Mnn}$ & 0.12 & 3.11 & 0.09 & 0.17 & 0.13 & 0.40 & 0.17 & 0.10 & 0.10 & 0.36 & 0.14 & 0.10 \\
\hline $\mathrm{Na}_{2} \mathrm{O}$ & 2.94 & 2.90 & 3.64 & 3.45 & 3.34 & 3.92 & 3.91 & 3.5: & 3.12 & 3.40 & 3.82 & 4.06 \\
\hline$k_{2} 0$ & 3.38 & 3.09 & 3.77 & 3.71 & 1.68 & 2,81 & 0.83 & 5.35 & 4.80 & 4.55 & 1.92 & 1.76 \\
\hline $\mathrm{P}_{2} \mathrm{C}_{5}$ & 0.32 & 0.28 & 0.41 & 0.29 & 0.23 & 0.33 & $\cong .33$ & 0.71 & 0.73 & 0.32 & 0.35 & $0.2 B$ \\
\hline $\mathrm{H}_{2} \mathrm{O}(+)$ & & & & & & & & & 0.44 & & & 0.40 \\
\hline$i_{2} 0(-)$ & & & & & & & & & 5.20 & & & 0.18 \\
\hline $\mathrm{CO}_{2}$ & & & & & & & & & 0.47 & & & 0.07 \\
\hline $8 \times 0$ & & & & & & & & & 0.17 & & & 0.11 \\
\hline sro & & & & & & & & & 0.12 & & & 0.07 \\
\hline ratal & 99.79 & 99.71 & 100.46 & 100.39 & 100.62 & 100.32 & 99.77 & 101.02 & 99.49 & 99.94 & 101.02 & 99.64 \\
\hline \multicolumn{13}{|c|}{ CIPW Marms (wt 8 ) } \\
\hline 0 & .72 & 2.68 & 0.68 & & 9.36 & 9.24 & 6.63 & 4.85 & 13.37 & 2.67 & 11.33 & \\
\hline \multicolumn{13}{|l|}{ io } \\
\hline Or & 19.97 & 18.26 & $\tilde{\varepsilon} 2.2 z$ & 4.20 & 9.93 & 16.61 & 4.90 & 31.61 & 28.36 & 26.89 & 11.29 & 10.40 \\
\hline$A D$ & 24.88 & 24.54 & 30.80 & 29.19 & 28.26 & 33.17 & 28.17 & 29.70 & 26.40 & 28.77 & 32.32 & 34.35 \\
\hline $4 n$ & 33.14 & 13.23 & 10.29 & 25.01 & 21.03 & 17.93 & 28.43 & 9.10 & 12.50 & 10.95 & 22.81 & 20.24 \\
\hline Me & & & & & & & 2.34 & & & & & \\
\hline$D \boldsymbol{i}$ & $: 3.25$ & 13.83 & 13.78 & 15.69 & 9.05 & 1.19 & 13.71 & 11.55 & 9.26 & 8.76 & 12.39 & $7.4 ?$ \\
\hline $\mathrm{Hy}$ & 19.40 & 19.50 & 9.44 & 6.84 & 15.45 & 9.47 & & 4.60 & B. 04 & 5.89 & i0.65 & 8.32 \\
\hline $0 i$ & & & & 13.10 & & & 13.30 & & & & & \\
\hline Mt & 5.63 & 5.22 & 4.20 & 3.19 & 5,26 & 4.26 & 3.57 & 4.15 & 4.52 & 3.22 & 5.32 & 4.22 \\
\hline $11 \mathrm{~m}$ & 2.05 & 1.65 & 2.03 & 2.51 & 1.75 & 1.59 & 3.99 & 2.03 & 2.09 & 1.37 & 2.77 & 1.82 \\
\hline \multicolumn{13}{|l|}{ tm } \\
\hline$A p$ & .76 & .56 & .97 & .69 & .54 & .73 & .78 & 1.68 & 1.73 & .76 & .83 & .66 \\
\hline Cc & & & & & & & & & 1.07 & & & .16 \\
\hline
\end{tabular}


TABLE II ícont\}

Picacho Butte Area

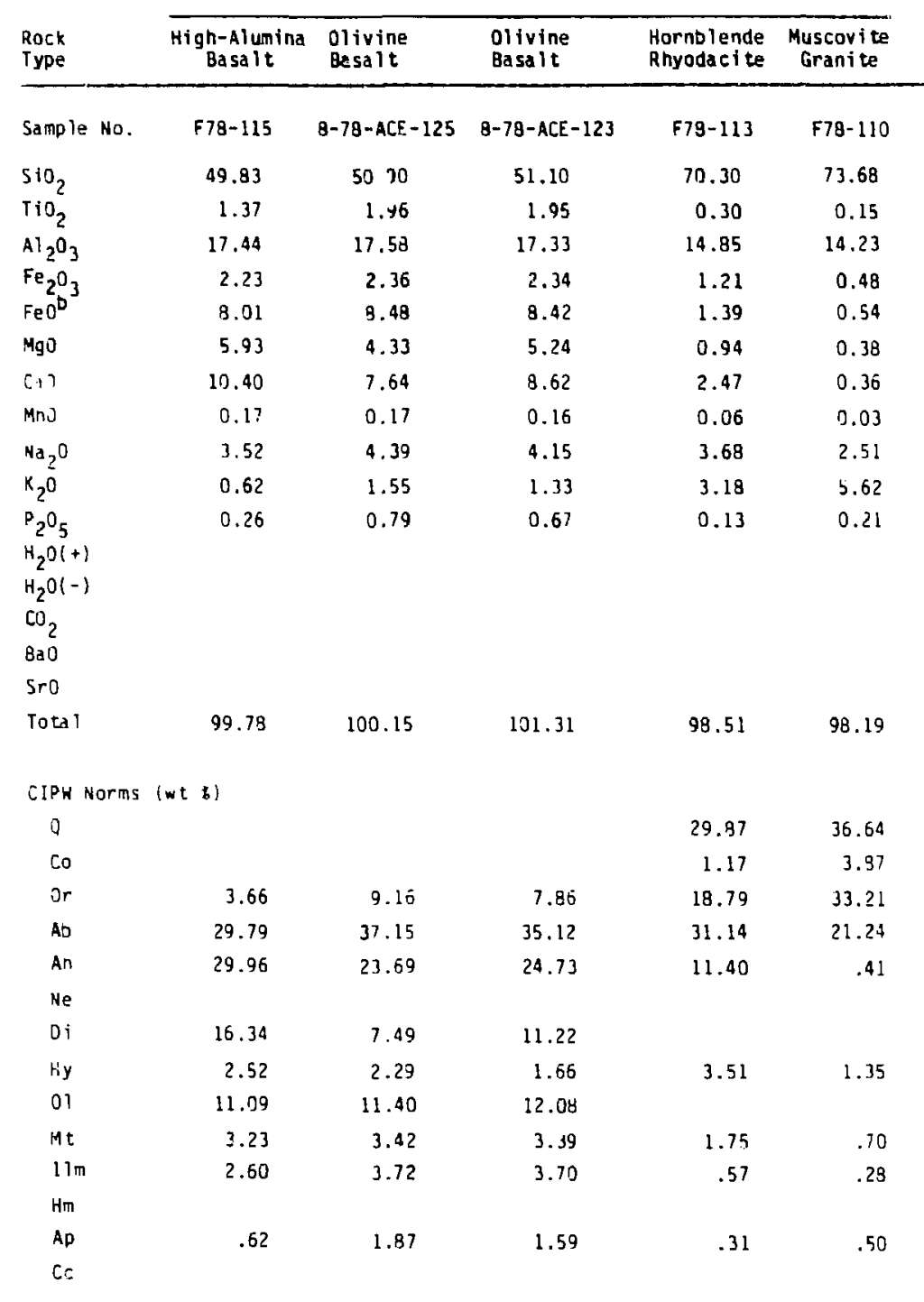

a Samples F78-45, F78-64,F79-83, F78-98, and 8A78-76 were wet chemically analyzed Dy J. Husler. Univ. of New Mexico; a 11 orer analyses (except those published by Fuis) are by $P$. Hooper. Washington state University, using XzF techn iques.

${ }^{\mathrm{O}} \mathrm{Fe}_{2} \mathrm{O}_{3}$ set at 0.20 total Fe $\left(1 \mathrm{~S} \mathrm{Fe}_{2} \mathrm{O}_{3}\right.$ ) for $\leq 53 \% \mathrm{SiO}_{2}$ and at 0.44 total Fe for

$\approx$ All rormalized to 1008 water free. 


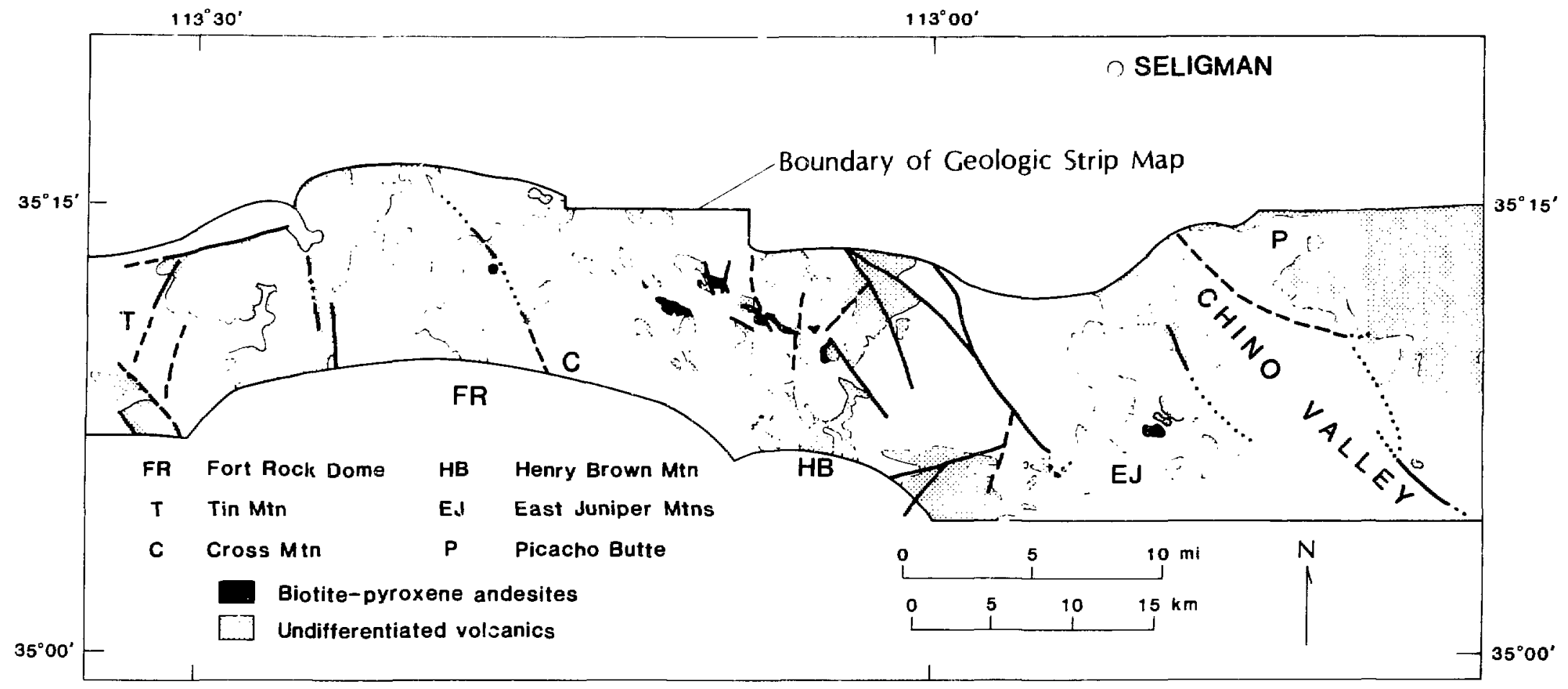

Fig. 2 .

Sketch of portion of strip map crossing transition zone. Volcanic units and locations mentioned in text are indicated. 


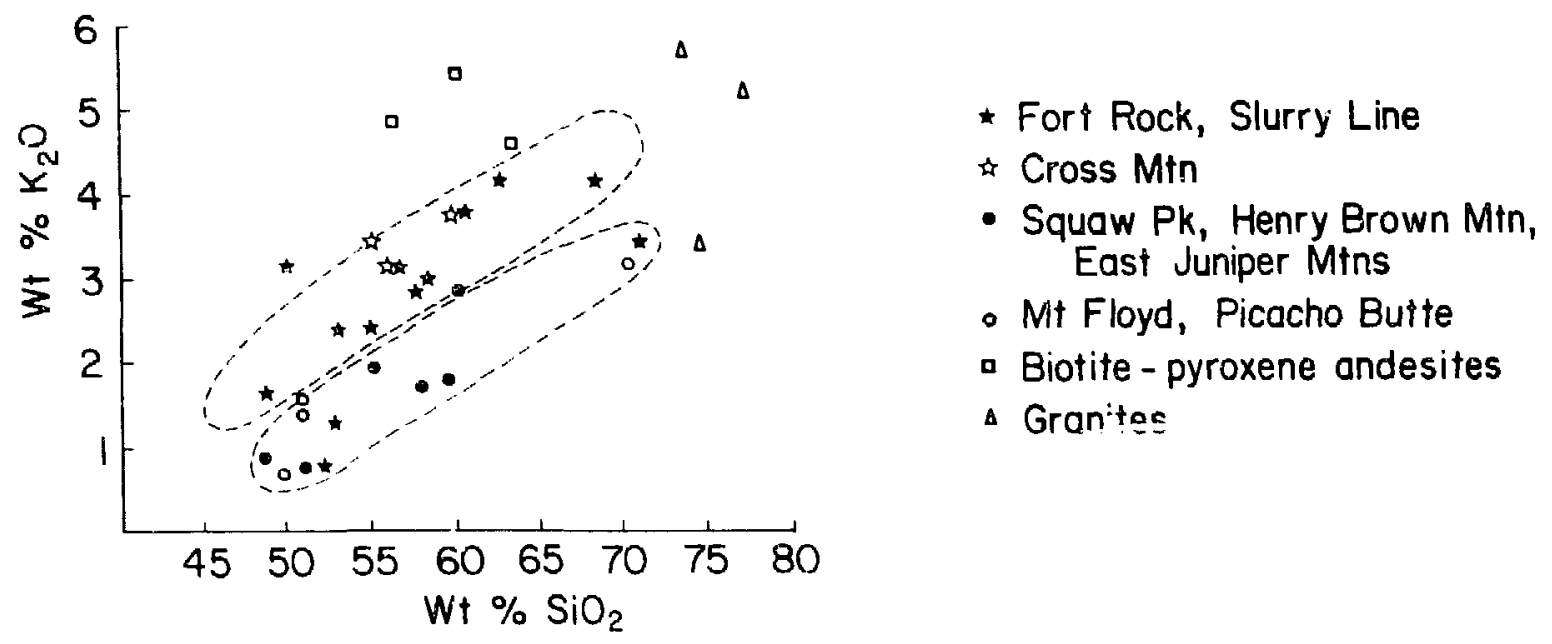

Fig. 3.

$\mathrm{K}_{2} \mathrm{O}$ vs $\mathrm{SiO}_{2}$ diagram showing eastern (low-potassium) and ${ }^{2}$ western (high-potassium) groups.

Peach Springs Tuff occurs north of Cross Mountain. The other tuff in this area is the Fort Rock Creek Tuff, equivalent to the Fort Rock Creek Rhyodacite of Fuis (1973), which underlies the Peach Springs Tuff. Peach Springs Tuff overlies most of the volcanics, but basalts and basaltic andesites in the North Fort Rock-Slurry Line area are younger than Peach Springs Tuff. Most of the Fort Rock volcanics are in the high-potassium group (Table II).

The Cros: Mountain basalte fall into the high-potassium group, but our one attempt to date this group was unsuccessful because the argon was $100 \%$ atmospheric. The age dates we obtained from Fort Rock volcanic rocks range in age from 24.3 to 20.7 Myr (Table I). Although Young and McKee (1978) obtained a date of 18.2 Myr on a basalt which underlies Fort Rock Creek Tuff, a basalt dike in the center of the Fort Rock area, which has cut and locally metamorphosed the Fort Rock Creek Tuff, was dated at 22.0 Myr. More dates are needed to resolve age-dating discrepancies and to better understand this complex volcanic area.

Precambrian rocks, mostly granite and granite gneiss, underlie the basalts in the Ft. Rock area. West of Cross Mountain, Cambrian sedimentary rocks overlie the Precambrian grarite. Cross Mountain itself is composed of limestone. Basalts overlie the limestone at lower elevations.

The eastern part of the transition zone contains volcanic rocks from the Squaw Peak, Henry Brown Mountain, and East Juniper Mountain areas. Each area 


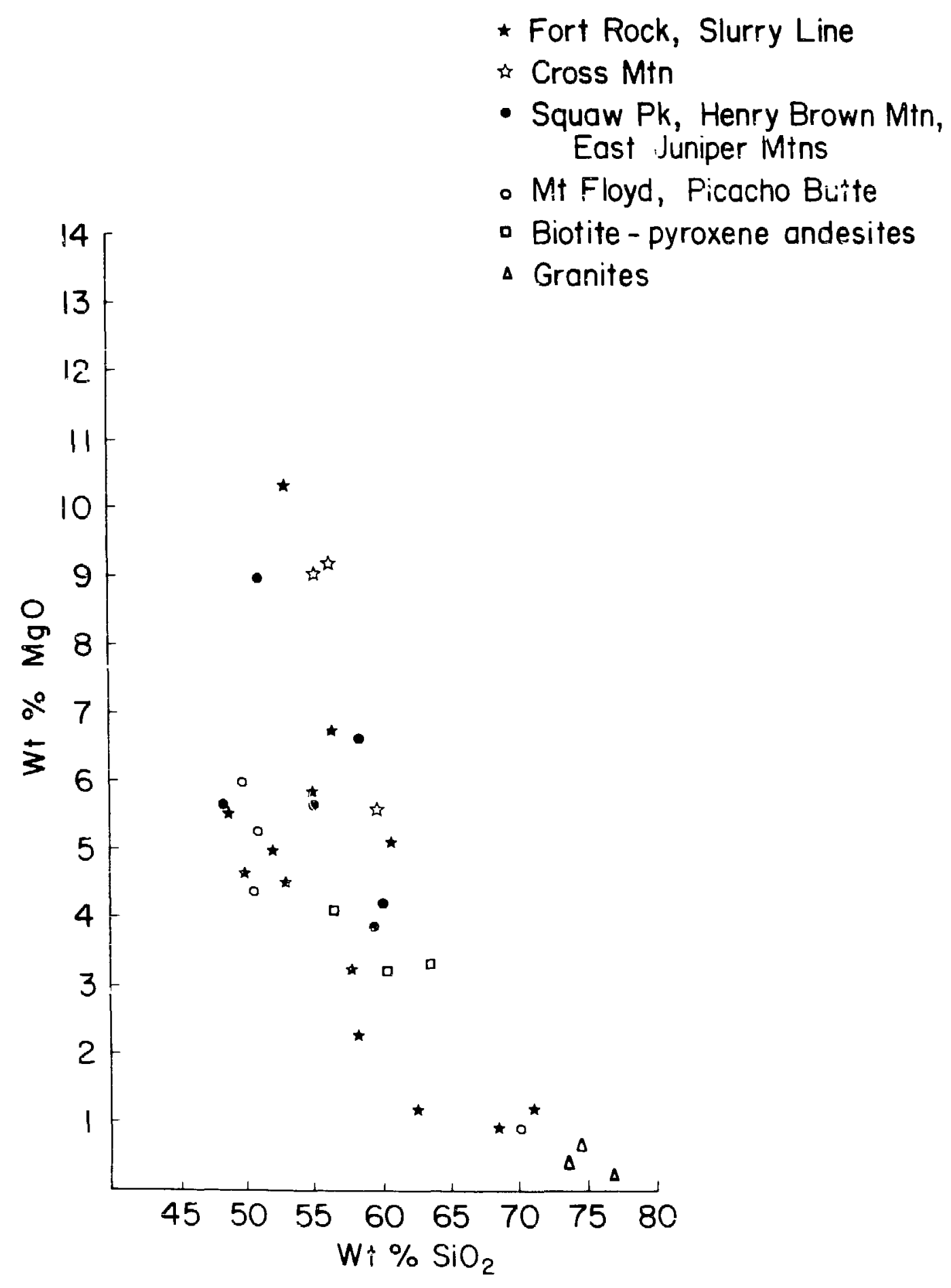

Fig. 4.

Harker variation diagrams for selected elements other than $k_{2} 0$. 


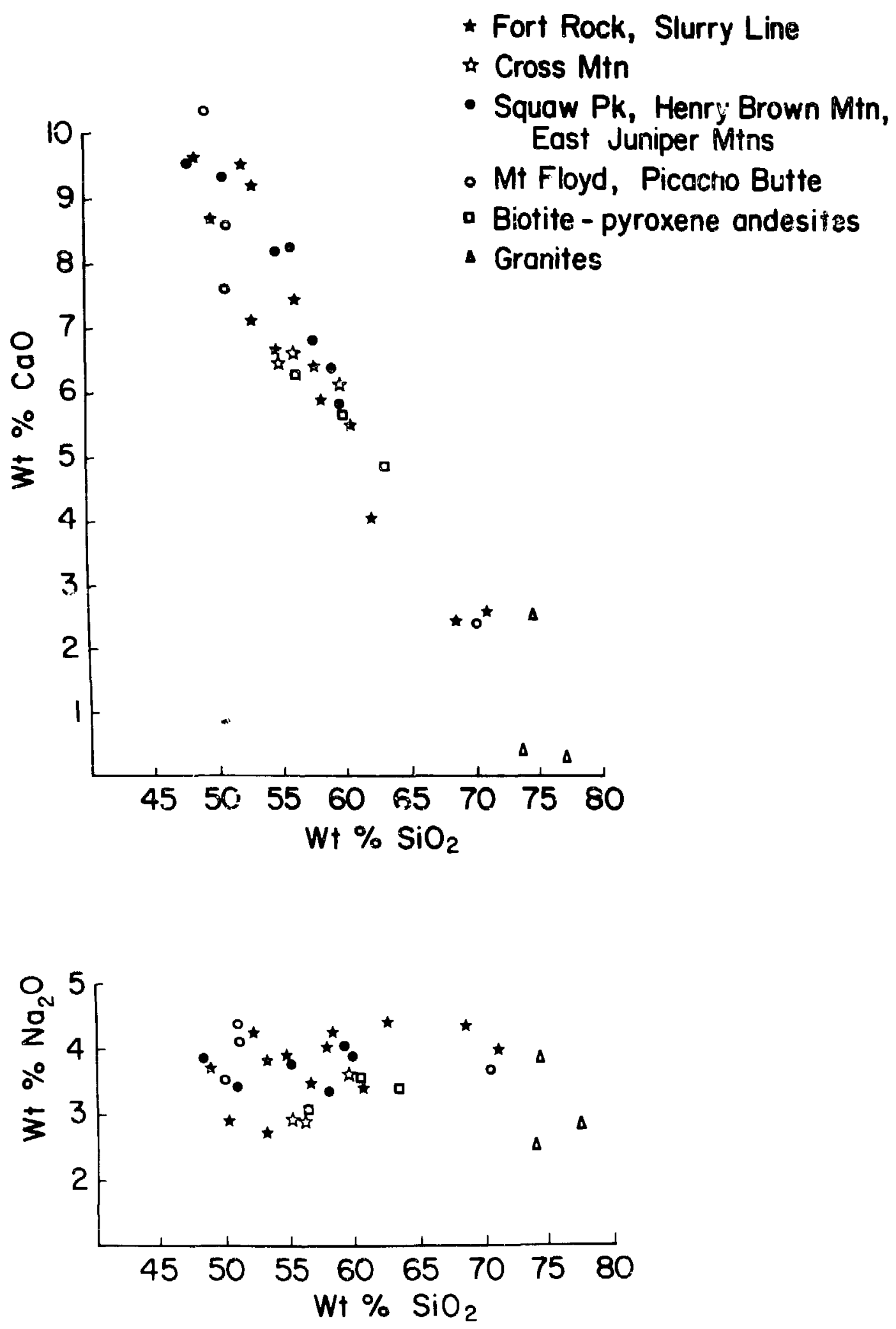

Fig. 4. (cont) 

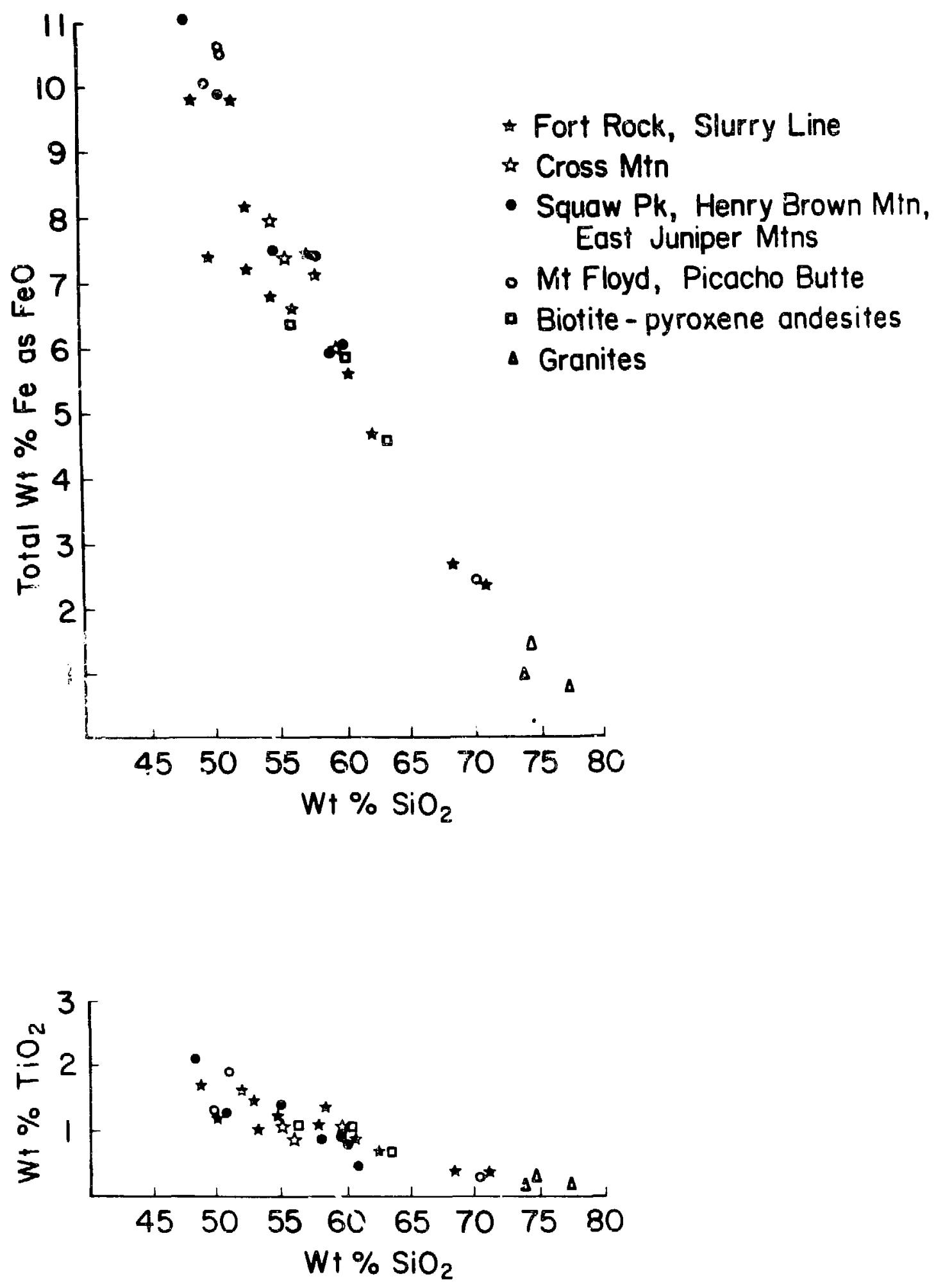

Fig. 4. (cont) 


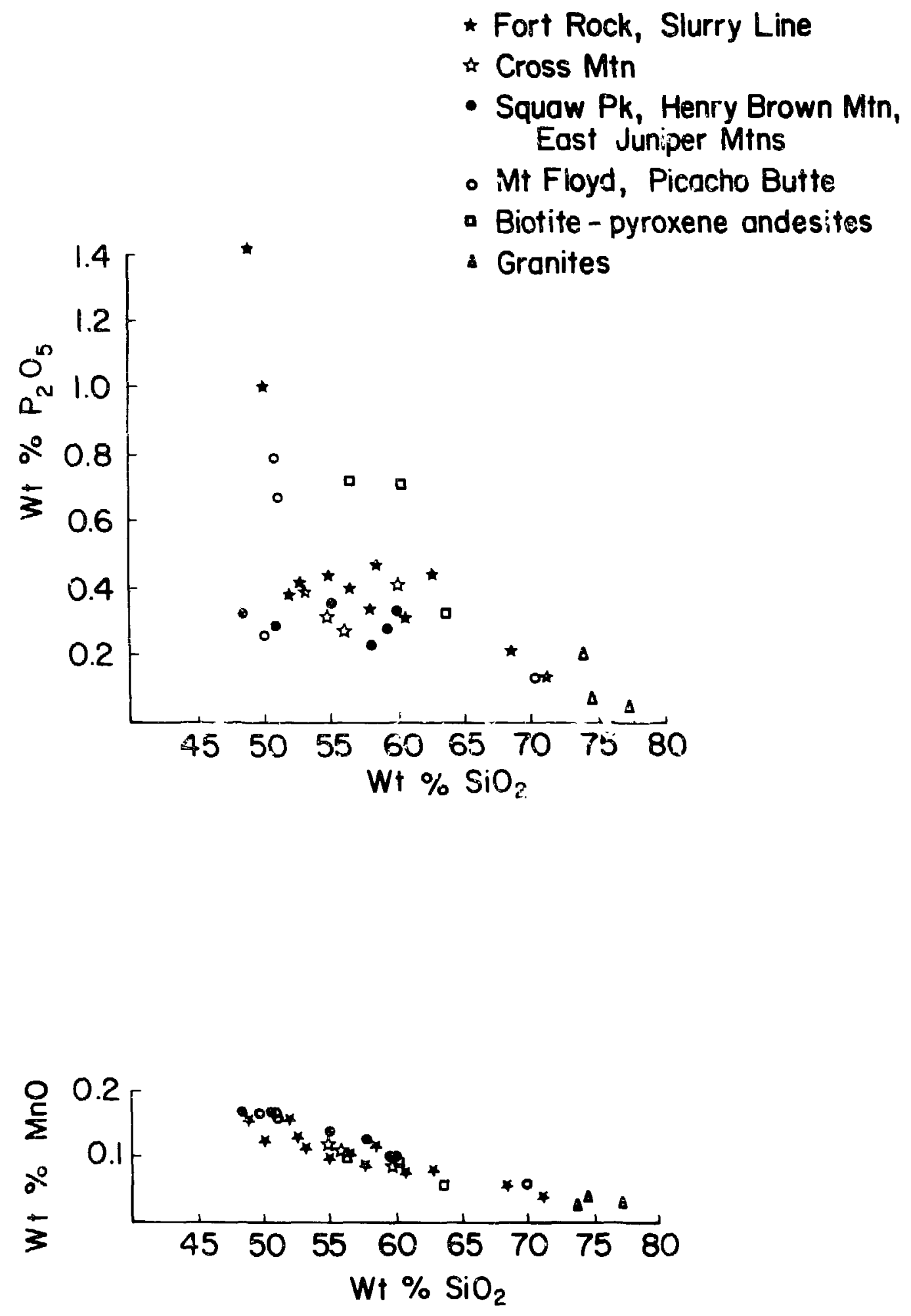

Fig. 4. (cont) 
- Fort Rock, Slurry Line

it Cross Min

- Squaw Pk, Henry Brown Mtn, East Juniper Mtns

- Mt Floyd, Picacho Butte

- Biotite - pyroxene anciesites

a Granites

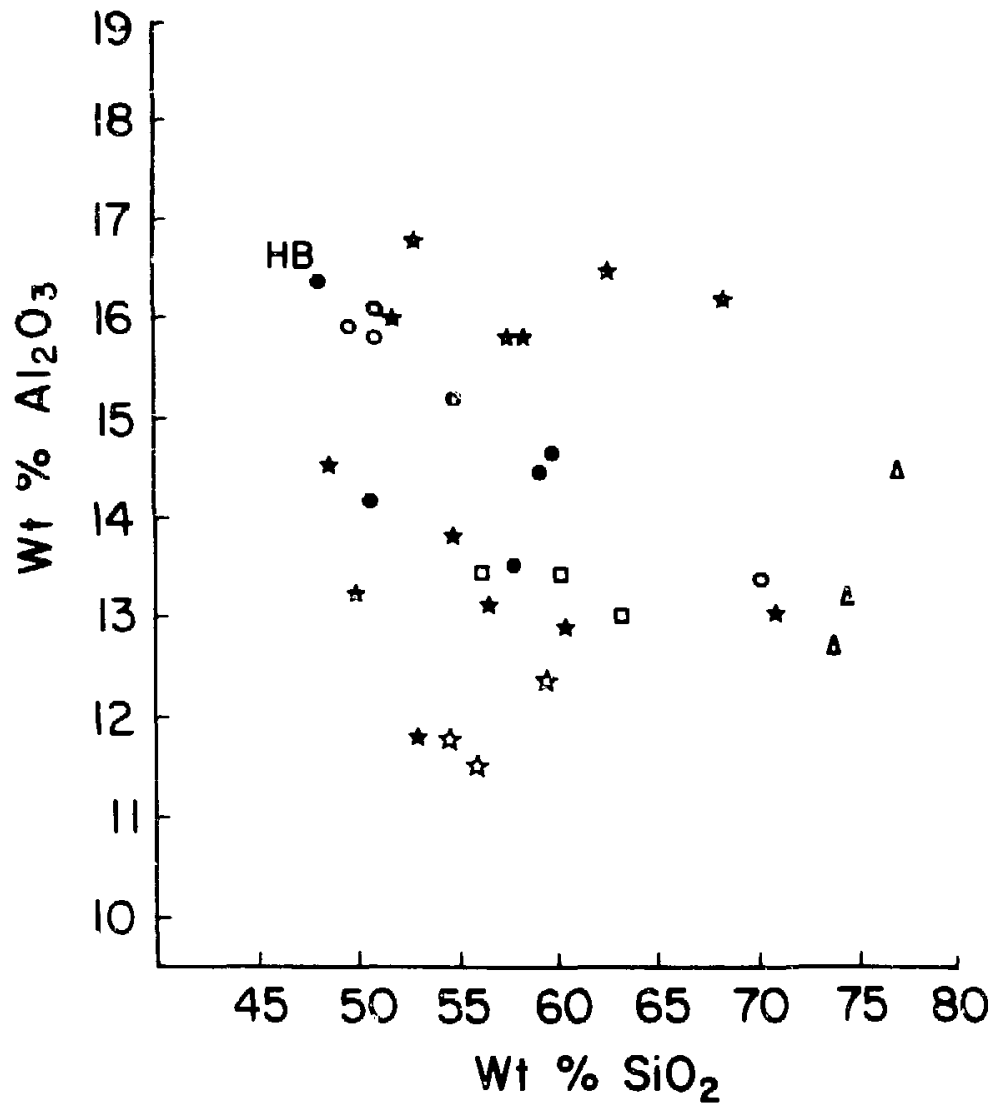

Fig. 4. (cont) 
within the group has its own sequences of basalt that are distinct petrographically and chemically. Squaw Peak basalts are tholeiitic and contain clusters of olivine phenocrysts. Henry Brown Mountain basalts are cosisegrained high-alumina basalts with dikcytaxitic texture. Th. East Juniper Mountain area has three types of basalt: olivine basalt, alkali basalt with abundant large phenocrysts of olivine, pyroxene, plagioclase, and a basaltic andesite with felted feldspar. The rocks from all these areas are grouped together by their lower frotassium contents and younger ages than the fort Rock-Cross Hountain volcanic rocks (Fig. 3 and Table I). There appears to be a gap between the ages of the youngest rocks in the Fort Rock area and the basalts of the eastern transition zone, but the oldest basalt in the eastern zone for which we obtained a date $(9.0 \mathrm{Myr}+1.2 \mathrm{Myr})$ is the youngest of the Squaw Peak eruptive series and the ages of the older units in the area are unknown. At present, there are too few dates to determine if the age gap is apparent or real.

Many basalts in all areas of the transition zone coritain resorbed quartz xenocrysts suggesting that crustal contamination may have played a part in the geriaration of these magnas. The scapolite phenocrysts in a latite from the cor Pock area and the similarity in the $\mathrm{K}_{2} \mathrm{O} / \mathrm{Na}_{2} \mathrm{O}$ ratio of meny volcanics and underlying granites also indicate a crustal component during generation of many intermediate to silicic melts. Unfortunately there are no isotopic or trace element data to better define petrogenetic relationships between the different rock types.

IV. MT. FLOYD VOLCANIC FIELD (EASTERN ZONE)

The Mt. Floyd-Picacho Butte area lies east of the Chino Valley and includes some flows just north of East Juniper Mountains. The Mt. FloydPicacho Butte volcanics fit the Eastern Transition Zone group chemically, but structurally the area is part of Colorado Plateau. The basalts are alkali olivine and alkali-rich high-alumina basalts. Just east of Chino Valley they have flowed around the rhyodacite dome of Picacho Butte. A date of $9.8 \mathrm{Myr}$ was obtained for this dome (Table I). Chemically and mineralogically it is similar to other silicic domes in the Mt. Floyd field (Nealey 1980) and may be the oldest and southernmost silicic dome of that group. The Mt. Floyd field is adjacent to and continuous with the San Francisco volcanic field. 


\section{SUMMARY}

A 10-to 15-km-wide strip was mapped crossing the transition zone between the Basin and Range on the west and the Colorado Plateau on the east. Chemical analyses and age dates were obtained for the volcanic rocks to aid in the mapping and to discern general trends in chemistry, time, and space. Volcanic rocks of the Basin and Range are basalt, basaltic andesite, andesite, dacite, and Peach Springs Tuff, a rhyolitic ash-flow tuff. Volcanic rocks overlie Precambrian granite, gneies and schist. A silicic volcanic field of sma 1? volume occurs in the Fort Rock area on the western part of the transition zone. Volcanic rocks include basalt, basaltic andesite, andesite, latite, dacite, a rhyodacite tuff, and Peach Springs Tuff. Ages on rocks underlying Peach Springs Tuff range from $20.7 \pm 0.6$ to $24.3 \pm 0.8$ Myr. Volcanic rocks in the eastern part of the transition zone are mostly basalt, and our dates range in age from $5.5 \pm 0.2$ to $9.0 \pm 1.2 \mathrm{Myr}$. An 01 der (s22 to 25 Myr) group of siotite-pyroxene andesites occurs along an $E-W$ band of unknown tectonic significance crossing the central transition zone. The volcanic rocks overlie Precambrian granite and granite gneiss in the west and $\mathrm{Pa}$ eoozoic sediments of increasing thickness in the eastern part of the transition zone.

The volcanic rocks in the Colorado Plateau Province are mostly basalts and basaltic andesites of the Mt. Floyd and San Francisco volcanic fields. A date of $9.8 \pm 0.7 \mathrm{Myr}$ obtained on a rhyodacite from Picasho Butte makes it the oldest rhyodacite complex in the Mt. Floyd-San Francisco Peak volcanic fields.

\section{ACKNOWIEDGGENTS}

We thank J. Husler and $P$. Hooper for chemical analyses and D. Krummenacher for $\mathrm{K}-\mathrm{Ar}$ age dates. Extended acknowledgments are given on the reconnaissance map. Thanks to D. Vaniman and D. Krier for review of this manuscript.

\section{REFERENCES}

Aiken, C. L. V., and M. E. Ander, "A Regional Strategy for Geothermal Exploration with Emphasis on Gravity and Magnetotellurics," J. Volcan. Geotherm. Res. 9, 1-27 (1981).

Ander, M. E., and R. B. Furgerson, "Magnetotelluric Study of Crustal Electrical Conductive Zones in the Southwestern U.S.," Abst., 6th Workshop on Electromagnetic Induction in the Earth and Moon. National Assoc. of Geomagnetism and Aeronomy 5.7 (1982). 
Ander, Mark E., "Correlation Between Deep Electrical Conductors and the Thermal State Within the Crust," Abst., EOS 64, p. 693 (1983).

Fuis, G., "The Geology and Mechanics of Formation of the Fort Rock Dome, Yavapai County, Arizona," Ph.D. Thesis, California Institute of Technology (1973).

Goff, F. E., B. H. Rirney, and A. C. Eddy, "Scapolite Phenocrysts in a Latite Dome, Northwest Arizona, USA," Earth Planet. Sci. Lett. 60, 86-92 (1982).

Goff, F. E., A. C. Eddy, and B. H. Arney, "Reconnaissance Geologic Map from Kingman to South of Bill Williams Mountain, Arizona," Los Alamos National Laboratory report LA-9202-MAP (1983).

Nealey, L. David, "Geology of Mount Floyd and Vicinity, Coconino County, Arizona," M.S. Thesis, Northern Arizona University (7980).

Young, R. A., and W. J. Brennan, "Peach Springs Tuff: Its Bearing on Structural Evolution of the Colorado Plateau and Development of Cenozoic Drainage in Mohave County, Arizona," Geol. Soc. Amer. Bul1. 85, 83-90 (1974).

Young, R. A., and E. H. McKee, "Early ant Middle Cenozoic Drainage and Erosion in West-Central Arizona," Geol. Soc. Amer. Bul1. 89, 1745-1750 (1978). 


\section{APPENDIX}

\section{PETROGRAPHIC DESCRIPTIONS OF SAMPLES IN TABLES I AND II}

\section{Kingman Area}

F78-50 0livine Basalt (Tkob):* Abundant olivine phenocrysts, slightly resorbed and iddingsitized along fractures and edges; phenocrysts of zoned clinopyroxene; fine-grained intersertal groundmass containing microlites of plagioclase, clinopyroxene and magnetite; vesicles filled with radiating, low birefringent minerals, probably silica and zeolites(?).

F78-45 Quartz-Bearing Basaltic Andesite (Tkqb): Euhedral phenocrysts of clinopyroxene and olivine altered to mixture of Fe-oxides, clays, and silica; euhedral phenocrysts and microphenocrysts of hornblende oxidized to opaque minerals; resorbed and complexiy zoned plagioclase phenocrysts; sparse resorbed quartz xenocrysts; trachytic groundmass consists of microcrystalline feldspar, clinopyroxene, hornblende, and glass; fractures and vesicles filled with carbonate.

F78-49 Quartz-Bearing Andesite (Tkqb): Resorbed phenocrysts and clots of hypersthene; sma 11 clots of hypersthene and plagioclase; contains resorbed quartz xenocrysts with fine-grained clinopyroxene reaction rims surrounded by haloes of plagioclase-hypersthene-glass; fine-grained intersertal groundmass of plagioclase, clinopyroxene and granular opaques; very little alteration.

Hualapai Mountains

F78-54 Syenogranite $(p \in g)$ : Very coarse-grained hypidionorphic-granular texture; contains rough!y $56 \%$ microcline, $32 \%$ quartz, $7 \%$ plagioclase, $3 \%$ opaques, and $1 \%$ biotite; biotite partly altered to chlorite; microcline partly altered to sericite; trace epidote along grain boundaries.

\footnotetext{
*Letters in parentheses refer to map symbols on LA-9202-MAP.
} 


\section{Peacock Mountains}

6-78ACE-16 0livine Andesite (Tkai): Euhedral pseudomorphs of olivine phenocrysts altered to Fe-oxides, clays, and sericitrif; abundant phenocrysts of clinopyroxene and altered phiogopite in unusuai groundmass of poikilitic-like feld:-; additional microlites of magnetite, ilmenite, and fres prilogopite.

Black Mesa

F78-64 Andesite (TDa): Phenocrysts and glomerophenocrysts of plagioclase in trachytic matrix of felted plagioclase, opaques, and very small clinopyroxene microphenocrysts.

F78-61 Biotite-01ivine Andesite (Tboa): Abundant phenocrysts of biotite, clinopyroxene, iddingsitized olivine, and rare phenocrysts of plagioclase; contains biotite-clinopyroxene clots. Glassy groundmass consists of microlites of clinopyroxene, biotite, and apatite.

F78-59 Hornblende-Biotite Dacite (Tbpd): Large phenocrysts of complexly zoned, resorbed plagioclase and resorbed quartz; some plagioclase shows sieve texture; smaller phenocrssts of resorbed hornblende and biotite in glassy groundmass containing hornblende, biotite and plagicclase microphenocrysts.

F78-60 Granite (pEg): Medium-grained hypidiomorphic-granular iexture; contains $40 \%$ quart7, $30 \%$ microcline and perthitic microcline, $23 \%$ plagioclase, $3 \%$ myrmekite, $3 \%$ spaques, and $1 \%$ muscovite + biotite; plagioclase altered partly to sericite; mica altered to chlorite.

Peach Springs Tuff

F78-63 Welded Rhyolitic Tuff (TPs): Phenocrysts of resorbed quartz, euhedra1 sanidine, and rare phenocrysts of euhedral plagioclase; contains sparse microphenocrysts of oxidized biotite, hornblende, clinopyroxene, sphene, and opaques; partially devitrified eutaxitic groundmass.

Fort Rock Area

6-78ACE-22 Fort Rock Creek Tuff (TfC): Reworked rhyolitic tuff containing ragged crystal fragments of quartz, K-feldspar, plagioclase, hornblende and biotite and rounded fragments of pumice and other volcanics; crystals make up nearly $50 \%$ of rock and are slightly layered; devitrified groundmass consists of glass shards and shard fragments; minor clay alteration. 
6-78ACE-30C 0livine Elasalt (Trob): Vesicular basalt containing severely iddingsitized olivine, minor clinopyroxene and opaque microphenocrysts; contains about $1 \%$ of biotite that may be dueteric or vapor-phase alteration; intersertal groundmass is extremely fine grained and composed of plagioclase, opaques, clinopyroxene and olivine; some vesicles filled with anaicime.

6-78ACE-35 0livine Basalt (Trob): Vesicular basalt having resorbed to euhedral phenocrysts of iddingsitized olivine, clinopyroxene, and plagioclase, and clots of these thiree phases in intersertal groundmass of plagioclase, clinopyroxene, olivine, opaques, and minor glass.

F78-80 Hornblende Andesite (Trha): Phenocrysts of resorbed oxidized hornblende and microphenocrysts of oxidized hornblende, minor plagioclase, and rare clinopyroxene; larger hornblende phenocrysts contain inclusions of clinopyroxene: groundmass is fine grained, devitrified, slightly vesicular, almost trachytic and contains microlites of plagioclase and opaques.

6-78ACE-37 Basaltic Andesite (Triba): Sparse phenocrysts of resorbed plagioclase and resoroed xenocrysts of quartz with clinopyroxene reaction rims in fine-grained pilotaxitic groundmass having microlites of plagioclase, clinopyroxene, and opaques and minor glass.

BA79-14 Hornblende Andesite (Trha): Phenocrysts of golden-yellow hornblende $(0.25$ to $1 \mathrm{~mm})(4.5 \%)$ with oxidized black rims, some are totally opaque; biotite $(0.5 \%)$, mostly oxidized opaque; sparse quartz and microcline fragments; very fine-grained pilotaxitic groundmass contains plagioclase laths $\sim 0.05 \mathrm{~m}(25 \%)$ and magnetite (6\%) in devitrified glass (64\%).

F78-74 Scapolite-Bearing Hornblende Latite $(\operatorname{Trh} 1)$ : Phenocrysts of resorbed to euhedral, complexly zoned plagioclase, hornblende, biotite, and scapolite; contains plagioclase clots and microphenocrysts of allenite and opaques; pilotaxitic groundmass contains microlites of plagioclase, hornblende, biotite, and devitrified glass.

BA78-30 Hornblende Rhyodacite (Trpd): Phenocrysts of elihedral hornblende $(5.5 \%)$, some zoned, and sparse biotite $!^{n} .5 \%$ ), both up to $1 \mathrm{~mm}$ long. Slightly to strongly resorbed, zoned plagioclase phenocrysts (10\%), mostly $\sim 1 \mathrm{~mm}$, but some to $2 \mathrm{~mm}$; granular groundmass contains stubby 
plagiociase (14\%), opaques, some of which may be biotite (0.5\%) anc biotite and amphibole $(0.8 \%)$ in devitrified glass.

F78-72 Hornblende Latite (Trh1): Phenocrysts of complexly zoned hornblende and minor biotite; contains rare granitic xenoliths; pilotaxitic groundmass contains plagioclase, hornblende, opaques and glass.

F78-85 01ivine Basalt (Trbi): Phenocrysts of iddingsitized olivine and clinopyroxene in glassy, vesicular, groundmass containing microlites of plagioclase, olivine, clinopyroxene, and opaques; contains small plagiorlase-clinopyroxene clots.

F78-87 Biotite Granite (pEg): Medium-grained hypidiomorphic-granular texture, locally sheared; contains roughly 40\% quartz, 25\% microcline, $25 \%$ plagioclase, $8 \%$ biotite, and $2 \%$ opaques, sphene, and others; sericite, epidote, and chlorite alteration particularly along crushed areas between biotite and plagioclase.

Nor th Fort Rock Area (Slurry Line)

F78-83 0livine Basalt (Tsb): Very porphyritic, roughly 20\% phenocrysts of olivine and clinopyroxene in glassy, vesicular, intersertal groundmass containing microlites of plagioclase, clinopyroxene and opaques; contains care xenocrysts of resorbed quartz; vesicles partly filled with carbonate.

BA78-37 0livine Basalt (Tsb): Slightly vesicular. Glomerocrysts of plagioclase to $3 \mathrm{~mm}$, and more rarely of plagioclase and clinopyroxene \pm olivine, phenocrysts of plagioclase, and of olivine with narrow iddingsitized rims. Intergranular groundmass with interstitial clinopyroxene, orange pseudomorphs after olivine(?) and skeletal opaques. Minor brownish alteration of plagiorlase in glomerocrysts.

F78-79 Quartz-Bearing Basaltic Andesite (Tsba): Phenocrysts of iddingsitized olivine and clinopyroxene and xenocrysts of quartz with clinopyroxene reaction rims; contains clots of fine-grained clinopyroxene; glassy intersertal groundmass contains microlites of plagioclase, clinopyroxene and opaques; some vesicles partly filled with carbonate.

BA78-89 01ivine-Hypersthene Andesite (TSa): Large resorbed phenocrysts of hypersthene occasionally rimmed with olivine or clinopyroxene and 
small phenocrysts and clots of clinopyroxene, iddingsitized olivine and rare plagioclase; contains resorbed xenocrysts of quartz and rare xenoliths of metamorphosed shale in vesicular hyalopilitic groundmass containing microlites of plagioclase, clinopyroxene and opaques.

BA78-73 Basalt (Tsb): Abundant phenocrysts, glomerocrysts and resorbed fragments of olivine (to $1.25 \mathrm{~mm}$ ) and clinopyroxene (to $2 \mathrm{~mm}$ ); rare inultigranular fragments of quartz with narrow kelphytic rims (of clinopyroxenel; hyalopilitic groundmass of clinopyroxene, plagioclase, and opaques in brown glass.

\section{Cross Mountain Area}

F78-89 Basaltic Andesite (Tcob): Abundant small phenocrysts of iddingsitized olivine and clinopyroxene; contains rare resorbed plagioclase phenocrysts and resorbed xenocrysts of quartz; glassy intersertal groundmass contains microlites of plagioclase, clinopyruxene and opaques.

8-78ACE-21 0livine Andesite (TCOa): Abundant pheriocrysts of iddingsitized olivine and clinopyroxene; contains rare sieve-textured plagioclase and clinopyroxene; contains very rare quartz with clinopyroxene reaction rims; intersertal to almost ophitic groundmass of clinopyroxene, plagiociase, and opaques; minor carbonate filling of vesicls.

F78-102 Biotite Andesite (Tcba): Phenocrysts of clinopyroxene and oxidized biotite; contains sieve-textured clinopyroxene and clinopyroxene clots; contains quartz xenocrysts with clinopyroxene reaction rims and very rare xenocrysts of granular quartz; intersertal to pilotaxitic groundmass of plagioclase, biotite, clinopyroxene and opaques.

Squaw Peak Area

BA78-52 01ivine Basa 1t (Tpob): Abundant large $(0.6$ to $3 \mathrm{~mm}$ ) phenocrysts and glomerocrysts of olivine rimmed with iddingsite; smaller 10.4 to $0.6 \mathrm{~mm}$ ) olivine phenocrysts are totally iddingsitized; trachytic groundmass with interstitial clinopyroxene and magnetite.

BA78-59 Quartz-Bearing Basaltic Andesite (Tpoi): Phenocrysts of resorbed iddingsitized olivine and smaller phenocrysts and clots of hypersthene; abundant xenocrysts of quartz with some reaction rims of 
fine-grained clincpyroxene; microphenocrysts of iddingsitized olivine and hypersthene; glassy pilotaxitic groundmass containing microlites of plagioclase, clinopyroxene, hypersthene and opaques.

BA78-63 Hornblende Andesite (Tpha): Abundant small (0.3 to $0.4 \mathrm{~mm}$ ) phenocrysts of hornblende rimed with opaque, biotite $(00.8 \mathrm{~mm})$ now almost totally opaque, larger very aitered (granular) clinopyroxene, sparse plagioclase, sparse quartz xenocrysts with kelphytic rims; groundmass of devitrified glass with plagioclase and rare incipient spherulites.

Henry Brown Mounta in

8-78ACE-14 High-Alumina Basa1t (Thb): Large phenocrysts of resorbed complexly zoned or sieve-textured plagioclase and resorbed iddingsitized olivine in coarse-grained diktytaxitic groundmass of plagioclase, olivine, clinopyroxene, opaques, and minor glass; vesicles contain minor carbonate.

Turkey Canyon Andesite ( $T$ tC)

F78-88 Biotite-Pyroxene Andesite: Phenocrysts of resorbed clinopyroxene, oxidized biotite, rare resorbed plagiociase, and prismatic apatite; contains biotite, apatite, clinopyroxene clots; contains rare xenocrysts of quartz; fine-grained pilotaxitic groundmass contains plagioclase, clinopyroxene, oxidizad biotite, and opáques.

F78-98 Biotite-Pyroxene Andesite: Phenocrysts and clots of clinopyroxene, severely oxidized biotites, and rare phenocrysts of plagioclase; contains large granular clinopyroxene-plagioclase cluts; piiotaxitic to almost trachytic groundmass of plagioclase, clinopyroxene, oxidized biotite, and opaques.

BA78-71 Biatite-Pyroxene Andesite: Abunciant phenocrysts and glomerocrysts of clinopyroxene and severely oxidized biotite; pilotaxitic groundmass of plagioclase, clincpyroxene, biotite, and opaques, and devitrified glass.

BA78-72. Biotite-Pyroxene Andesite: Abundarit phenocrysts of biotite (up to $4 \mathrm{~mm}$, but commonly $1 \mathrm{~mm}$ ) and less abundant phenocrysts of clinopyroxene (to $1 \mathrm{~mm}$ ), some glomerocrysts of both; phenocrysts of pyroxene often have inclusions of biotite; larger biotites contain inclusions of clinopyroxene; biotite is oxidized orange but retains original birefringence; contains sparse hornblende; pilotaxitic 
groundmass contains interstitial hornblende(?) microlites and microphenocrysts of magnetite in devitrified glass.

East Juniper Mountains Area

BA78-82 Pyroxene Basalt ( $T \mathrm{jpb}$ ): Large (to $3 \mathrm{~mm}$ ) phenocrysts of corroded plagioclase, slightly resorbed olivine with thin rims of iddingsite, and clinopyroxene; intersertal groundmass of plagioclase, clinopyroxene, opaques, and olivine.

BA78-76 Basaltic Andesite ( $T$ jba): Small phenocrysts (to $1 \mathrm{~mm}$ ) of plagioclase and microphenocrysts of hypersthene and olivine; pilotaxitic groundmass contains plagioclase, clinopyroxene, and opaques.

BA78-90 0livine Basalt (Tjob): Large (to 3 min) phenocrysts of corrodeci plagioclase, some with sieve texture, and resorbed and embayed olivine with narrow rims of iddingsite. Intersertal groundmass with microphenocrysts of plagioclase and olivine in devitrified glass containing anhedral clinopyroxene and abundant microgranular opaques.

Picacho Butte Area

F78-115 Hign-Alumina Basalt ( $T f \circ b):$ Small phenocrysts of iddingsitized olivine and plagioclase in medium-grained diktytaxitic groundmass of plagioclase, clinopyroxene, olivine, opaques, and glass.

8-78ACE-125 0livine Basalt (Tfob): Phenocrysts of complexly zoned to sieve-textured plagioclase, iddingsitized olivine, anc ophitic clinopyroxene in trachytic groundmass of plagioclase, clinopyroxene, olivine, and opaques; contains small clinopyroxene-plagioclase clots. 8-78ACE-123 0livine Basalt ( $T f \circ b)$ : Phenocrysts of iddingsitized olivine and microphenocrysts of plagioclase in pilotaxitic groundmass of plagioclase, clinopyroxene, olivine, and opaques.

F78-113 Hornblende Rhyodacite (Tfhd): Phenocrysts of biotite, hornblende, plagioclase, and $\mathrm{K}$-feldspar in glassy vesicular pilotaxitic groundmass containing microlites of plagioclase, hornblende, bioti te, and opaques.

F78-110 Muscovite Granite $(p \in g)$ : Medium-grained hypidiomorphic granular texture; contains quartz, microcline and some perthite, plagioclase, muscovite and rare biotite and opaques; plagioclase altered to sericite; nicas contain some chlorite alteration. 